\title{
Litotripsia extracorpórea por ondas de choque en niños. A propósito de un caso complicado
}

\author{
Dr. Rafael Alvarado-García, ${ }^{*}$ Dr. Alejandro Xicoténcatl Mundo-Alegría **
}

\section{RESUMEN}

La litotripsia extracorpórea es un procedimiento eficaz en el tratamiento de litiasis renal y ureteral. En pediatría ha adquirido gran aceptación por la facilidad del procedimiento, mayor disponibilidad de litotriptores y acortamiento de la estancia hospitalaria. Sin embargo, las complicaciones que puede desarrollar el paciente pediátrico pueden variar desde las sintomáticas (dolor) hasta las que comprometen la función renal. Presentamos el caso de una adolescente de 15 años de edad con litiasis renal bilateral que fue sometida a una sesión de litotripsia extracorpórea. Esta intervención causó un hematoma renal izquierdo, que se visualizó con ultrasonido y tomografía computada renal. Se trató en forma conservadora y el hematoma desapareció 13 semanas después. Cuatro semanas después la paciente desarrolló hipertensión arterial sistémica; hubo disminución de la tasa de filtración glomerular en el riñón ipsilateral que cedieron posteriormente. La litotripsia extracorpórea en pediatría es eficaz para el tratamiento de litiasis renal y ureteral. Es un procedimiento sencillo y rápido. El desarrollo de un hematoma renal es una complicación infrecuente, menor al 1\%. La monitorización y el seguimiento clínico del paciente permiten identificarla y tratarla oportunamente.

Palabras clave: Litotripsia, litiasis, hematoma renal, hipertensión arterial.

\section{ABSTRACT}

Extracorporeal shock wave lithotripsy is an effective procedure for the treatment of renal and ureteral lithiasis. In pediatrics has become widely accepted by the facility of the procedure, availability of lithotripters and faster discharge from hospital. However, complications in pediatric patients can vary from symptomatic (pain) to a compromise renal function. We present a case of a 15 year old female adolescent with bilateral renal lithiasis which underwent for extracorporeal shock wave lithotripsy session which was complicated by a left renal hematoma, confirmed by renal ultrasonography and computerized tomography scan. Conservative management was established and the hematoma became stabilized. The patient developed hypertension and glomerular filtration rate was decreased on the ipsilateral kidney four weeks after the procedure which had spontaneous resolution. Pediatric extracorporeal shock wave lithotripsy is an effective procedure for the treatment of renal and ureteral calculi; its advantages are easiness, speed and accessibility. A renal hematoma as a complication occurs in less than $1 \%$. Patient monitoring and follow-up identified timely this complication.

Key words: Lithotripsy, lithiasis, renal hematoma, hypertension.

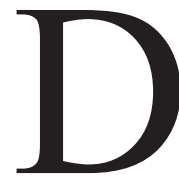

esde 1980 Chaussy y colaboradores ${ }^{1}$, realizaron la primera litotripsia extracorpórea en Alemania. La extracción de cálculos mediante cirugía abierta ha disminuido considerable-

* Servicio de Urología Pediátrica

** Servicio de Cirugía Pediátrica

CMN 20 de Noviembre, ISSSTE

Correspondencia: Dr. Rafael Alvarado-García. CMN 20 de Noviembre, ISSSTE. Félix Cuevas No. 540. Col. del Valle. México 3100 D.F.

Recibido: junio, 2009. Aceptado: febrero, 2010.

Este artículo debe citarse como: Alvarado-García R, Mundo-Alegría AX. Litotripsia extracorpórea por ondas de choque en niños. A propósito de un caso complicado. Acta Pediatr Mex 2010;31(3):95-101.

www.nietoeditores.com.mx mente. En la actualidad la litotripsia extracorpórea y la endourología son el tratamiento de primera elección para la litiasis renal. El primer informe del uso de litotripsia extracorpórea en niños lo realizaron Newman y colaboradores en $1986^{2}$. En los años noventa del siglo XX aparecieron numerosas publicaciones acerca de la efectividad y seguridad a corto y largo plazo del tratamiento de la litiasis renal en niños.

Aunque es un procedimiento no invasivo, no es inocuo, por lo que hace necesario conocer las complicaciones inmediatas, mediatas y tardías. Anteriormente se recomendaba proteger el pulmón para evitar el trauma a ese órgano ${ }^{3-5}$. En la actualidad se recomienda la protección durante la litotropsia de cálculos ureterales bajos en niñas, para evitar el daño a los ovarios ${ }^{3,6}$. 
El desarrollo de hematoma renal como complicación del uso de litotripsia extracorpórea no es frecuente; es menor al $1 \%{ }^{5}$. Presentamos el caso de una adolescente con litiasis renal sometida a una sesión de litotripsia extracorpórea por ondas de choque (LEOCH), con litotriptor electromagnético quien desarrolló un hematoma renal. Comentamos generalidades de la litotripsia, su aplicación en niños y sus complicaciones.

\section{CASO CLÍNICO}

Adolescente femenina de 15 años de edad, originaria de Jalapa, Veracruz. Había antecedentes heredofamiliares de litiasis renal en la madre y en la abuela materna. Tuvo infección de vías urinarias en dos ocasiones, acompañado de dolor abdominal de tipo cólico a nivel de ambos flancos, irradiado a región lumbar de predominio izquierdo que cedió temporalmente con analgésico y antibiótico (ketorolaco y trimetroprim con sulfametoxazol por 15 días). Posteriormente tuvo dos cuadros similares con intervalo de dos meses; en el último evento tuvo vómito de contenido gástrico. Exploración física. Peso y talla adecuados para la edad (porcentila 50), Giordano izquierdo positivo, dolor a la palpación media en fosa iliaca e hipocondrio izquierdo, peristaltismo disminuido sin órganomegalias aparentes.

Laboratorio: Biometría hemática: hemoglobina: 12.7 $\mathrm{g} / \mathrm{L}$; hematócrito: $37 \%$; leucocitos: $6,500 \mathrm{cel} / \mathrm{mm}^{3}$; neutrófilos, $65 \%$; linfocitos, $32 \%$; monocitos, 2\%; TP: 12.9 (92\%), TPT 30.4. Na, $133 ; \mathrm{Cl}, 110 ; \mathrm{K}, 4.5$; creatinina sérica, $0.72 \mathrm{mg} / \mathrm{dL}$.

En la radiografía de abdomen se identificaron imágenes radiopacas de bordes irregulares, dentro de ambas siluetas renales. El ultrasonido renal reveló datos de hidronefrosis bilateral leve e imágenes hiperecoicas en ambas pelvis renales que proyectan sombra acústica, sugestivas de litiasis renal bilateral, la izquierda con diámetro de $7 \mathrm{~mm}$ x $5 \mathrm{~mm}$ y la derecha de $5 \mathrm{~mm}$ x $4 \mathrm{~mm}$. La pielografía ascendente mostró adecuado paso del medio de contraste a ambos ureteros y defectos de llenado a nivel de ambas pelvis renales, datos que corroboraron una litiasis renal bilateral. Gammagrama renal con ácido dietilentriaminopentaacético (DTPA por sus siglas en inglés), adecuada función renal bilateral. Se le dio tratamiento con analgésicos y antimicrobianos por 15 días y se programó para litotripsia extracorpórea.
Mediante fluoroscopia se identificó un cálculo en el riñón izquierdo a nivel del cáliz renal, de unos $20 \mathrm{~mm}$ de diámetro. Bajo analgesia y sedación se realizó LEOCH mediante litotriptor electrohidráulico. Se aplicó un kilovoltaje de 14 a 18 con un total de 3000 disparos con lo que se fragmentó el cálculo. Dos horas después la paciente desarrolló dolor progresivo en la región lumbar izquierda, persistente aún con tratamiento analgésico, hematuria macroscópica progresiva y vómito de contenido gástrico en cuatro ocasiones. Exploración física: Temperatura $37^{\circ} \mathrm{C}$, frecuencia cardiaca $90 / \mathrm{min}$, tensión arterial $130 / 100 \mathrm{~mm} / \mathrm{Hg}$; palidez de tegumentos, mucosa oral ligeramente seca. Se corroboró dolor abdominal en cuadrante inferior derecho a la palpación media y profunda; no había ruidos peristálticos. Biometría hemática postlitotripsia: Hemoglobina: $8.7 \mathrm{~g} / \mathrm{L}$; hematócrito, $30 \%$; leucocitos, $13,200 \mathrm{cel} / \mathrm{mm}^{3}$; neutrófilos, $75 \%$; linfocitos, 22\%. Plaquetas, 135 mil. Otro ultrasonido renal mostró una imagen hiperecoica fuera del parénquima renal izquierdo de $8 \times 8 \mathrm{~cm}$ en sus diámetros mayores, escaso líquido libre perirrenal izquierdo sin deformación de la pelvis renal ni los cálices renales. Una tomografía axial computada simple mostró un hematoma subcapsular de 5.5 x $9.2 \mathrm{~cm}$ que comprimía en forma importante el parénquima renal izquierdo (Figura 1). Se le trató mediante ayuno, soluciones parenterales, transfusión de concentrado eritrocitario (20 mililitros por kilogramo, dosis total), analgésico, antiespasmódico intravenoso y reposo absoluto. Evolucionó favorablemente. A las 24 horas se inició la alimentación por vía oral. El dolor abdominal disminuyó y la hematuria remitió al cuarto día con corrección de la anemia aguda en la biometría hemática. Un ultrasonido renal de control al mes mostró un hematoma residual del riñón izquierdo de

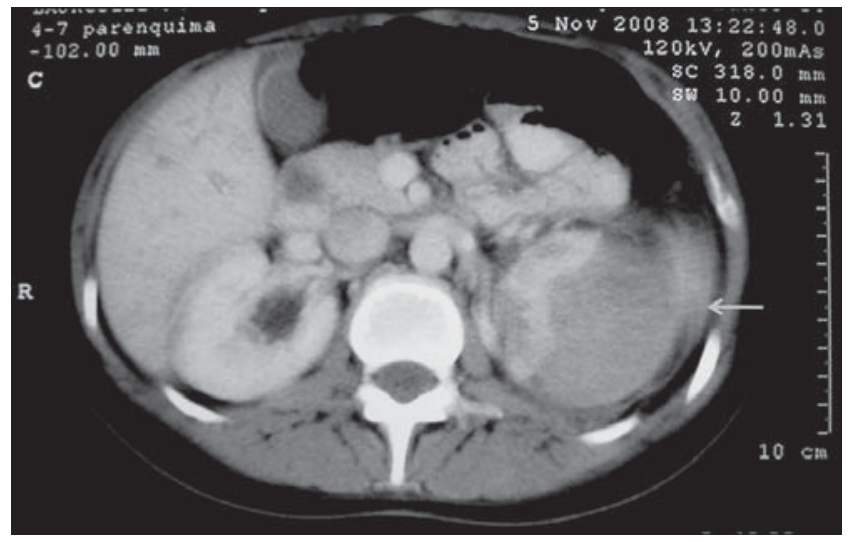

Figura 1. Tomografía abdominal con contraste y hematoma renal (flecha). 
69 x $26 \mathrm{~mm}$, con volumen aproximado de $35 \mathrm{cc}$; un cálculo residual de $3 \times 7 \mathrm{~mm}$, el cual remitió a los tres meses y que se vio en los ultrasonidos de control.

La paciente tuvo hipertensión arterial asintomática por unas ocho semanas después de la litotripsia. No hubo que administrar medicamentos; la tensión arterial se normalizó a las doce semanas. El último gammagrama renal con MAG-3 y diurético mostró adecuada función renal bilateral. Se descartaron causas metabólicas, anatómicas e infecciosas que pudieran generar litiasis renal en esta paciente, por lo que se consideró de índole idiopático. Se vigila a la paciente por consulta externa trimestralmente y se valora la función renal con gammagrama renal y ultrasonido renal cada seis meses.

\section{DISCUSIÓN}

La frecuencia de litiasis renal en nuestro país es mayor a lo señalado en la literatura internacional. Esto se debe a la dureza del agua para consumo humano en algunas regiones de México ( $\geq 400$ partículas por mililitro) y por los antecedentes heredofamiliares de litiasis renal ${ }^{7}$, siendo positivo por línea materna en el presente caso.

En los EE.UU. la prevalencia de litiasis renal en niños varía de 1 en 1,000 a 1 en 7,600 admisiones hospitalarias, dependiendo del área geográfica ${ }^{8}$, con una frecuencia de 3 al 5\% ${ }^{9}$. El 20\% de los casos de litiasis ureteral en niños es de escolares y lactantes. Predomina el sexo masculino con una relación de 1:4 ${ }^{10}$.

La litotripsia extracorpórea de ondas de choque (LEOCH) es eficaz en el tratamiento de la litiasis pieloureteral. Su empleo reduce los costos, el tiempo de hospitalización y el riesgo quirúrgico; es de fácil realización y no requiere anestesia general en la mayoría de los casos. La disponibilidad de los equipos se ha incrementado desde que se usó el primer litotriptor en México en $19877^{7}$. Actualmente se dispone de este recurso en los hospitales de tercer nivel (privados y de población abierta), pero los hospitales pediátricos de referencia e investigación carecen de éstos.

Se usa la LEOCH a partir de la etapa de lactante mayor y sus ventajas en el niño consisten en mayor eficacia en la desintegración de los cálculos, mayor aclaramiento de fragmentos residuales, movilización temprana del paciente y menor número de sesiones de LEOCH debido a que los cálculos tienen menos tiempo de haberse formado ${ }^{9}$. Es mayor la recurrencia en la formación de cálculos en niños en comparación con los adultos (44\% niños vs 8 a $10 \%$ adultos); sin embargo, la etiología compleja en este grupo (alteraciones metabólicas, malformaciones anatómicas e infecciones recurrentes), permite que la transmisión de las ondas de choque se facilite por el mayor contenido de agua en los tejidos del niño, por la menor resistencia al paso de la onda ${ }^{5,9,11}$.

Cuando la onda de choque llega a un cálculo, encuentra una resistencia elevada, por lo que parte de la onda se refleja, parte es absorbida y parte se transmite. El choque entre zonas de distinta impedancia acústica produce en la zona anterior del cálculo una fuerza de compresión y en la zona posterior se produce una fuerza de tensión con un rebote parcial de la onda inicial; la onda reflejada se superpone a la siguiente con un efecto de suma que aumenta sustancialmente las presiones en el interior del cálculo. Esta onda reflejada se denomina onda de tracción, la cual, al oponerse a la onda de presión provoca la fisura o fragmentación del cálculo ${ }^{12}$. A este mecanismo y a las propiedades acústicas entre los cristales y la matriz se ha atribuido a la ruptura en forma de fragmentos esféricos (sparring) de la parte posterior del cálculo y a la separación en capas de cebolla en el interior del mismo ${ }^{4}$. Por el contrario, en la parte anterior del cálculo se produce una erosión por cavitación: al originar distintos gradientes de presión, se forman burbujas de vapor de agua, que se expanden rápidamente y posteriormente se colapsan súbitamente y liberan ondas de choque de alta presión que explotan sobre la superficie ${ }^{13}$. (Figura 2)

Los cálculos de oxalato cálcico y cistina, tienen mayor impedancia acústica y son los más resistentes a la frag-

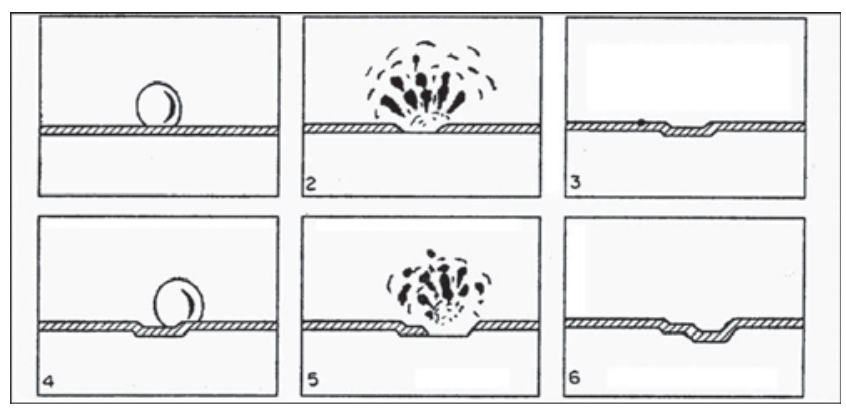

Figura 2. Cavitación. Cuando un líquido fluye a través de una región donde la presión es menor que su presión de vapor, el líquido hierve y forma burbujas de vapor. Estas burbujas son transportadas por el líquido hasta llegar a una región de mayor presión, donde el vapor regresa al estado líquido de manera súbita con implosión brusca de las burbujas, provocando daño en la pared de estructuras cercanas. 
mentación por esta técnica pues la energía que se refleja es mayor; los cálculos de estruvita y de fosfato cálcico apatita, que dejan pasar mejor la onda de choque y los más frecuentes en pediatría, se fragmentan más fácilmente ${ }^{12}$.

Existen diversos generadores de ondas de choque. El litotriptor piezoeléctrico es el ideal en pediatría ya que la onda de choque es de 2 a $3 \mathrm{~mm}$ y es suficientemente intensa para fragmentar los cálculos. Se recomiendan 1000 a 2000 disparos con un kilovoltaje de 12 a 14. Principales características de los litotriptores:

A) Litotriptor electrohidráulico (Figura 3). De primera generación, de la empresa Dornier, primer aparato de aplicación clínica (HM3). La onda de choque se genera por la acción de un electrodo que produce una intensa descarga eléctrica en un medio líquido (agua) y crea ondas por la vaporización que causa, y que se reflejan de forma divergente en una semielipse metálica (reflector de onda); esto permite reorientarlas a la zona del cálculo (lito) ${ }^{13}$. Tiene alta capacidad de desintegración pero causa mucho dolor por lo cual es necesario anestesiar al paciente ${ }^{5}$.

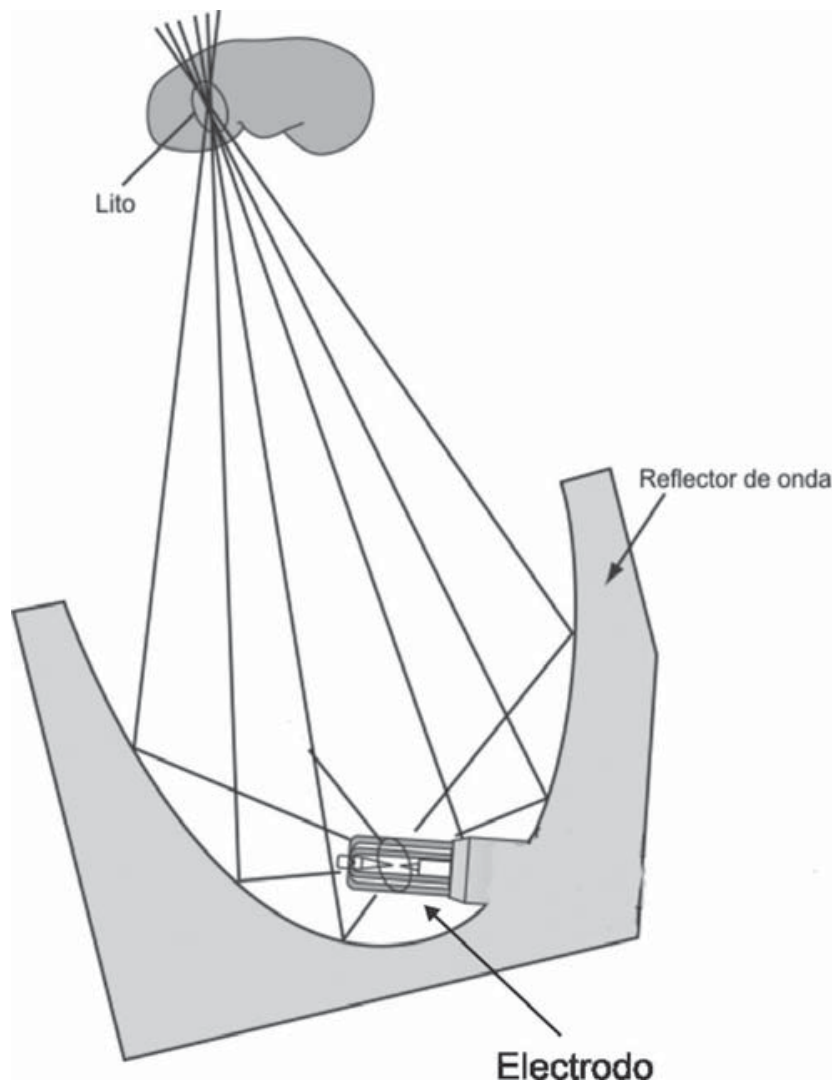

Figura 3. Litotriptor electrohidráulico (primera generación).
B) Litotriptor piezoeléctrico (Figura 4). De segunda generación, utiliza la energía piezoeléctrica y produce una onda de choque al pasar una corriente eléctrica por unas bandejas semiesféricas cubiertas de cristales piezoeléctricos cerámicos alineados, que induce presión elevada en un pequeño punto focal. La onda causa poco dolor y es suficiente para fragmentar cálculos. Sólo requiere analgesia y su punto focal de impacto es de 1 a $2 \mathrm{~mm}$, lo que lo hace ideal para su uso en pediatría. Su desventaja es que la fuente de energía tiene gran diámetro y en ocasiones son necesarias varias sesiones para fragmentar de cálculos en adultos ${ }^{5}$.

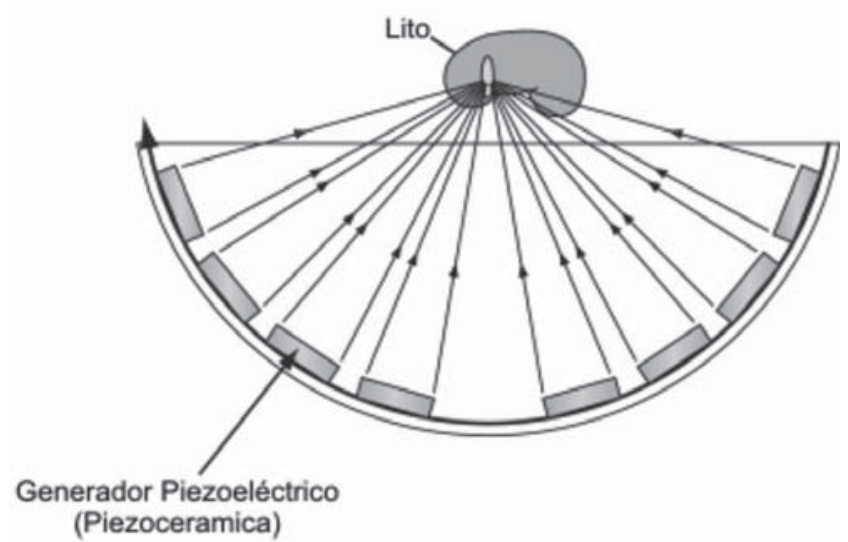

Figura 4. Litotriptor piezoeléctrico (segunda generación).

C) Litotriptor electromagnético. (Figura 5). De tercera generación. Su acción se basa en el desplazamiento retrógrado de una membrana metálica colocada dentro de un tubo donde una bobina plana genera una corriente magnética al ser excitada por una descarga eléctrica. La membrana metálica está cargada eléctricamente del mismo signo que la bobina; al pasar la corriente magnética, se desplaza con un movimiento brusco de repulsión, lo que genera presión suficiente para formar la onda de choque. La focalización se hace por lentes acústicas o reflector parabólico metálico ${ }^{1,4}$. La onda de choque es constante y la energía es dirigida a un pequeño punto focal con alta energía ${ }^{5}$.

Las ondas de choque se transmiten desde el punto de origen hasta el cuerpo por un medio líquido o semilíquido de baja impedancia acústica a fin de que la pérdida de energía sea la menor posible. Inicialmente este medio era un recipiente con agua donde se sumergía al paciente (HM3). En las nuevas máquinas se ha sustituido por cabezales llenos de agua y cubiertos por gel transmisor acústico que se sitúan en la zona a tratar. 


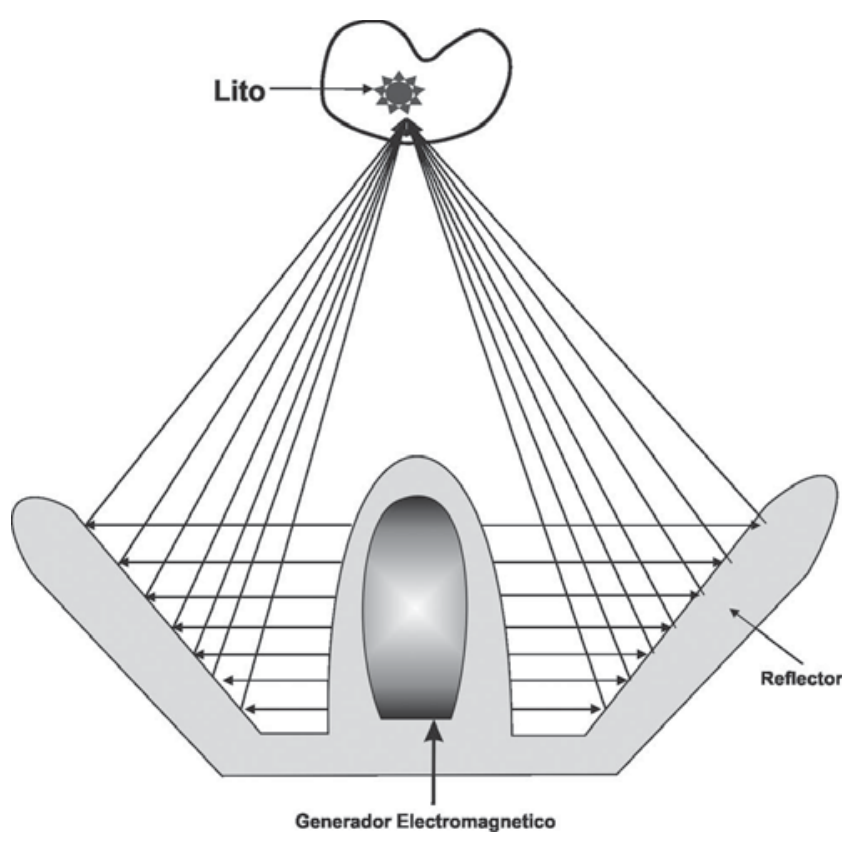

Figura 5. Litotriptor electromagnético (tercera generación).

\section{Complicaciones}

La LEOCH no es inocua; el empleo de ondas de choque no tiene efectos indeseables agudos o crónicos. Numerosas publicaciones afirman que la litotripsia extracorpórea no causa problema sobre el organismo; sin embargo, produce complicaciones hasta en $18 \%$ de los pacientes, algunas inmediatas como dolor cólico, síndrome neurovegetativo, fiebre, hematuria, urosepsis, dolor abdominal inespecífico, obstrucción ureteral, hematoma subcapsular, perirrenal e intrarrenal. Las complicaciones tardías son hipertensión arterial sistémica, fibrosis perirrenal y daño de la función renal ${ }^{14,15}$ (Cuadro 1).
La formación de un hematoma provocado por la litotripsia es una complicación infrecuente $(<1 \%)$. Su localización habitual es subcapsular como en nuestra paciente; ocasionalmente puede ser perirrenal. En niños los principales factores que predisponen a las complicaciones son: tipo de litotriptor; el uso de energía demasiado elevada; número excesivo de ondas de choque en cada sesión; intervalo insuficiente entre las sesiones de litotripsia, que debe ser entre 36 y 48 horas.

La onda de presión negativa es la causa del daño tisular y de los hematomas renales; la lesión se explica por el fenómeno de cavitación, que ocurre por la imposibilidad de la mayoría de los fluidos de soportar grandes presiones negativas, lo que afecta sobre todo a los pequeños vasos venosos del parénquima ${ }^{16}$. En nuestra paciente el tipo de litotriptor, la intensidad de la onda y el número de golpes pudieron haber causado el hematoma renal; se descartaron coagulopatías, uso de fármacos, alteraciones anatómicas o infección asociada.

El hematoma causa dolor lumbar persistente, vómito e irritabilidad; que no se debe al cólico por la expulsión de fragmentos litiásicos. La persistencia de sintomatología urinaria (disuria, pujo, tenesmo vesical, etc.) después de una LEOCH requiere hospitalizar y monitorizar al paciente; hacer un ultrasonido renal para descartar otro tipo de complicaciones, como se hizo en nuestra paciente. Los síntomas vegetativos (piel fría, mareos, sudación, etc.), hematuria macroscópica e inestabilidad hemodinámica con descenso brusco de hemoglobina/hematócrito también deben hacer pensar en esta complicación ${ }^{17}$.

Después de la litotripsia, el hematoma renal se diagnostica por ultrasonido en $0.6 \%$ de los pacientes; con la tomografía la frecuencia se eleva hasta en $25 \%{ }^{18}$.

Cuadro 1. Complicaciones de la litotripsia extracorpórea

\begin{tabular}{lccc}
\hline Complicaciones & Shouman 2009 & Grippo2002 & Arrabal 2000 $^{21}$ \\
\hline Hematuria macroscópica & $100 \%$ & $100 \%$ & $100 \%$ \\
Cólico renal & $8.1 \%$ & $8.7 \%$ & $28 \%$ \\
Fiebre & $2.1 \%$ & $2.4 \%$ & $1.1 \%$ \\
IVU & $4.7 \%$ & $6.5 \%$ & $0.1 \%$ \\
Dolor abdominal & & $0.4 \%$ & $0.4 \%$ \\
Hematoma renal & $0.1 \%$ & $0.19 \%$ & $0.4 \%$
\end{tabular}


La mayoría de los hematomas son pequeños y remiten espontáneamente, por lo cual el uso de la TAC se reserva para los casos con sintomatología persistente.

La lesión que causan las ondas de choque es semejante a las de un traumatismo renal externo: edema intersticial, ruptura de la vía urinaria (pelvis, uretero, etc.) y sangrado parenquimatoso hasta en $63 \%$ de los casos ${ }^{19}$. Los grandes hematomas renales y perirrenales causados por la LEOCH, son una complicación inmediata y potencialmente grave ${ }^{20}$. El tratamiento de un hematoma subcapsular generalmente es conservador: analgésicos, control estrecho de creatinina sérica y hematócrito en serie. Si este tratamiento no es suficiente y el paciente no se estabiliza, se recurre a la cirugía abierta ${ }^{1,13,18}$. La experiencia muestra que la posibilidad de perder un riñón cuando se hace necesario operar es elevada. Otra opción es efectuar un drenaje clásico con cirugía abierta a través de una lumbotomía o por laparoscopia ${ }^{21}$. Se ha descrito también la colocación de drenaje percutáneo ${ }^{21}$.

Existe controversia sobre el drenaje del hematoma en los casos que se mantienen estables; por un lado se dice que mejora la evolución y disminuyen los riesgos de secuelas como hipertensión arterial. Por otro lado, en algunos casos el hematoma puede dificultar la expulsión de fragmentos litiásicos (por compresión y distorsión de la vía urinaria); al drenar el hematoma se ha observado la expulsión de fragmentos algunas horas después. Sin embargo, no hay suficientes estudios para aseverar esto ${ }^{20}$. Después de un episodio agudo de hematoma subscapular renal, con cálculos residuales, se debe aplicar una nueva sesión de LEOCH, después de un periodo de espera al menos de tres semanas posterior al cuadro agudo ${ }^{13,18}$.

En nuestra opinión es recomendable el uso de litotriptor piezoeléctrico, en niños ya que tienen un punto focal de impacto de 1 a $2 \mathrm{~mm}$, la intensidad de las ondas de choque es menor y hay buen resultado sobre los litos más frecuentes en esta población.

El litotriptor electromagnético tiene puntos focales menores y mayor intensidad de la onda de choque, lo que eleva el riesgo de daño a la vasculatura renal. El inconveniente de los litotriptores de primera generación (hidroeléctricos) es que tienen áreas de impacto mucho mayores.

Si la función renal no se afecta el pronóstico de los pacientes es bueno, pero requiere un adecuado seguimiento clínico y por estudios de imagen, principalmente el ultra- sonográfico. La valoración nefrológica es imperativa para descartar alteraciones metabólicas que causen la formación de cálculos.

\section{CONCLUSIONES}

La litotripsia extracorpórea es un procedimiento eficaz en el tratamiento de la litiasis renal. La cirugía abierta debe ser una última opción. El riesgo de formación de hematomas post LEOCH es bajo. La monitorización del paciente y el uso del ultrasonido en caso de persistir con sintomatología postlitotripsia están indicados para descartar lesiones graves. Cuando hay litos residuales con o sin complicaciones graves como hematomas, se realizan dos sesiones más de LEOCH para la fragmentación de los cálculos. Cuando persiste el cuadro existen nuevas técnicas como el uso de láser de Holmio, pielolitotripsia percutánea y endoscópica.

Es recomendable el seguimiento estrecho a largo plazo de pacientes con litiasis renal y el estudio metabólico, anatómico-funcional de la vía urinaria es obligatorio para comprender el origen y desarrollo en la formación de litos.

\section{BIBLIOGRAFÍA}

1. Chaussy C, Schuller J, Schmiedt E, Brandl H, Jocham D, Liebl B. Extracorporeal shock-wave lithotripsy (ESWL) for treatment of urolithiasis. Urology. 1984;23:59-66.

2. Newman DM, Coury T, Lingeman JE, et al. Extracorporeal shock wave lithotripsy in children. J Urol. 1986;136:238-40.

3. Lottman H, Archambault F, Traxer O, Mercier Pageyral B, Hellal B. The efficacy and parenchymal consequences of extracorporeal shock wave lithotripsy in infant. $\mathrm{Br} \mathrm{J}$ Urol. 2000;85:311-5.

4. Ruiz Marcellan FJ. Litotripsia extracorpórea por ondas de choque. En: Actualización en el diagnóstico y tratamiento de la litiasis urinaria. $5^{\circ}$ Seminario de formación continuada en urología. Madrid: Ed. Ergon; 2000. p. 19-30.

5. D’Adessi A, Bongovianni L, Sasso F, Gulino G, Falabella R, Bassi P. Extracorporeal shockwave lithotripsy in pediatrics. A safe procedure? J Endourol. 2008;22(1):1-11.

6. Al Basaidy SS, Prem AR, Medhat M, Giriraj D, Gopakumar P, Bhat HS. Pediatric ureteric calculi: efficacy of primary in situ extracorporeal shock wave lithotripsy. Br J Urol. 1998;82:906.

7. Medina-Escobedo M, Zaidi M, Real-de León D, OrozcoRivadeneyra S. Urolithiasis prevalence and risk factors in Yucatán, Mexico. Salud Pub Mex. 2002;541:545.

8. Kroovand RL. Pediatric urolithiasis. Urol Clin North Am. 1997;24:173-84. 
9. Shultz-Lampel D, Lampel A. The surgical management of stones in children. BJY Inter. 2001;87:732-40.

10. Basaklar AC, Kalen N. Experience with childhood urolithiasis, report of 196 cases. Br J Urol. 1991;67:203-5.

11. Torrecilla-Ortíz C, Matías-López JJ, Contreras-García J, Aguiló-Lució F, Capms-Lloveras N, Riera-Canals L. Hematomas renales tras litotripsia extracorpórea por ondas de choque. Actas Perol Esp. 1977;21(8):752-57.

12. Zhong $P$, Preminger $G$. Physics of shock-wave lithotripsy. EN: Kidney stons. medical and surgical management. Coe F, Favus M, Pak C, Parks J, Preminger G. Editores. Filadelfia: Lippincott-Raven Publishers; 1996. p. 530-1.

13. Reina RM, Sánchez-de la Vega J, Martínez RR, Blasco HP, García PM. Litotripsia extracorpórea por ondas de choque. Un tratamiento establecido. Actas Urol Esp. 2002;26(9):636-49.

14. Skolarikos A, Alivizatos G, De la Rosette J. Extracorporeal shock wave lithotripsy 25 years later: complications and their prevention. Eur Urol. 2006;50(5):981-90.

15. Bhatta DN, Thornton J, Karafa MT, Streem SB. A multivariate analysis of risk factors associated with subcapsulas hematoma formation following electromagnetic shock wave lithotripsy. J Urol. 2004;172:2271-74.
16. Collado SA, Huguet PJ, Monreal GF, Rousaud BA, Izquierdo TF, Vicente RJ. Renal hematoma as a complication of extracorporeal shock wave lithotripsy. Scand J Urol Nephrol. 1999;33(3):171-5.

17. Rubin Jl, Arger PH, Pollack HM, Banner MP. Kidney changes after estracorporeal shock wave lithotripsy: CT evaluation. Radiology. 1987;162:21-9.

18. Pastor NP, Carrion LP, Martínez RJ, Pastor GJM, Martínez MM, Vsrseda RJA. Hematomas renales tras litotripsia extracorpórea por ondas de choque (LEOCH). Actas Urol Esp. 2009;33(3):296-303.

19. Gallego SJA, Ibarlucea GG, Gamarra QM, Guisasola J, Bernuy MC. Renal hematomas alter extracorporeal lithotripsy with the lithotriptor "lithostar multicine de Siemens". Actas Urol Esp. 2000;24(1):19-22.

20. Gram CW, Lynch SC, Muskat PC, Mokulis JA. Laparoscopic evacuation of a subcapsular renal hematoma causing symptomatic hypertension. J Endourol. 1998:12(6):551-3.

21. Jang YB, Kang KP, Lee S, Kim YG, et al. Treatment of subcapsular hematoma, a complication of extracorporeal shock wave lithotripsy, by percutaneous drainage. Nephrol Dial Transplant. 2006;21(4):1117-8. 